\title{
Determining PML Modes in 2-D Stratified Media
}

\author{
Szabolcs B. Gaál, Member, IEEE, Hugo J. W. M. Hoekstra, and Paul V. Lambeck
}

\begin{abstract}
A contour-integration method is presented to determine the eigenmodes in a layered structure closed with PML layers at the boundaries of the computational window. Improvements are provided to ensure the accuracy of contour integration and to assure that all the encircled eigenvalues are determined. Numerical examples are presented to test the accuracy of the method.
\end{abstract}

Index Terms-Contour integration, eigenmodes, perfectly matched layers (PMLs), slab waveguides, wave propagation.

\section{INTRODUCTION}

$\mathbf{T}$ HE MODAL expansion method (MEM) provides a powerful tool to describe the propagation of an input field through a layered two-dimensional (2-D) structure. In the design of integrated optical devices, it can be used to analyze an open-wave guiding system, which may vary in the $z$ direction (splitters, gratings, etc.). MEM also possesses the advantage of giving detailed information on the fraction of power traveling in the guided and radiation modes of the wave-guiding system at each longitudinal coordinate.

In the MEM, the discretization of the continuum of the radiation and evanescent modes is usually accomplished by applying an electric wall "sufficiently far" from the analyzed structure. The unwanted reflections from these walls can be prevented by introducing artificial absorptive layers, the perfectly matched layers (PMLs) at the boundaries of the computational window [1]. The great advantage of PMLs is, that by means of them we can eliminate the undesired reflections occurring at the edges of the computational window and thus mimic the open system.

Finding PML modes can be a hard task, since the effective indices belonging to these modes are complex. The methods used up to now to determine the PML modes use a sort of follow-up technique: having determined the eigenmodes for non-PML setting with standard methods, the eigenvalues are followed as they move in the complex plane due to gradually increasing the strength of the PMLs [2]. This method may be quite cumbersome in some cases, for example to determine the quasidegenerate system modes of a directional coupler and may eventually lead to losing of eigenvalues.

The problem of losing roots can be circumvented by the method of contour integration (CI) [3] However, when implementing the existing CI algorithms, we experienced instability and/or numerical inaccuracy for some cases. This paper presents improvements and hints to evaluate the CI for

Manuscript received February 11, 2002; revised August 12, 2002. This work was supported in part by Stichting Technische Wetenschappen, Utrecht, The Netherlands, under project STW-TOE.5071.

The authors are with the Lightwave Devices Group, Department of Applied Physics, MESA + Research Institute, Universiteit Twente, Enschede, The Netherlands.

Digital Object Identifier 10.1109/JLT.2003.808636

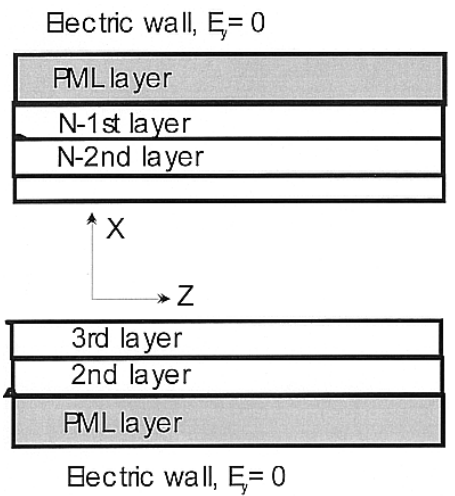

Fig. 1. Schematic picture of a typical structure. The top and bottom layers are PMLs. At the computational boundaries, the electric field is zero. The refractive indices of the layers are piecewise constant.

obtaining the effective indexes of the PML modes in a reliable way.

The paper is organized as follows. In Section II, we present expressions related to the CI method together with our imposed innovations related to a robust implementation. In Section III, a brief description is given on the computer program with an emphasis on the choices of input parameters. In Section IV, an inner product for eigenfunctions, a prerequisite for modal field expansion, is introduced. In Section V, we present numerical examples to test the accuracy of the method, to check whether reflections occur on the PMLs, and to test the accuracy of finding quasidegenerate eigenvalues. The paper ends with conclusions.

\section{CI METHOD}

In this section, we derive the required formulas and introduce the improvements for robust evaluation of the CI for structures with PMLs. We will consider that TE and TM polarization can be treated similarly.

\section{A. The Dispersion Relation in the Presence of PMLs}

The cross section of a typical structure is shown in Fig. 1. In the calculation, it is convenient to introduce a local coordinate system with $x=0$ at the beginning of each layer.

The modal field equation to be solved is

$$
\frac{1}{1-j \hat{\sigma}} \partial_{x} \frac{1}{1-j \hat{\sigma}} \partial_{x} E_{y}+\left(k_{0}^{2} n^{2}-\beta^{2}\right) E_{y}=0
$$

also obeying the Dirichlet boundary conditions $E_{y}=0$ at the computational boundaries. In (1), $\beta$ is the propagation constant, $k_{0}(\equiv 2 \pi / \lambda)$ the wavenumber, $n$ the piecewise constant refractive index, and $\hat{\sigma}$ represents the strength of the PMLs. Since the refractive indices are piecewise constant both in the wave- 
guiding layers and in the PMLs, the solutions of (1) take the form of $A_{+/-} \exp \left[ \pm \sqrt{(1-j \hat{\sigma})^{2}\left(\beta^{2}-k_{0}^{2} n^{2}\right)} x\right]$. The determination of the unknown amplitudes is considered later in Section IV of this paper. Using the continuity of $E_{y}$ and its derivative at the dielectric interfaces as well as the solution of (1), taking the aforementioned form, one can derive the following transfer matrix for TE polarized waves connecting the field and its derivative at the bottom of layer $p$ to that of layer $p+1$

$$
\begin{aligned}
{\left[\begin{array}{c}
E_{y, p+1} \\
\partial_{x} E_{y, p+1}
\end{array}\right]=} & \left(\begin{array}{cc}
\cos \left(\eta_{p} \alpha_{p} d_{p}\right) & \frac{\sin \left(\eta_{p} \alpha_{p} d_{p}\right)}{\alpha_{p}} \\
-\alpha_{p} \sin \left(\eta_{p} \alpha_{p} d_{p}\right) & \cos \left(\eta_{p} \alpha_{p} d_{p}\right)
\end{array}\right) \\
& \cdot\left[\begin{array}{c}
E_{y, p} \\
\partial_{x} E_{y, p}
\end{array}\right] .
\end{aligned}
$$

Here, $\eta_{p}=1-j \sigma$, with $\sigma \neq 0$ in PMLs and equal to zero otherwise, $d_{p}$ is the thickness of the $p$ th layer, and $\alpha_{p} \equiv \sqrt{\beta^{2}-k_{0}^{2} n_{p}^{2}}$. Note that the choice of the sign of the square root does not change the transfer matrix given in (2). By applying (2) for all layers, starting with

$$
\left[\begin{array}{c}
E_{y, 1} \\
\partial_{x} E_{y, 1}
\end{array}\right]=\left[\begin{array}{l}
0 \\
1
\end{array}\right]
$$

at the lower computational boundary, the electric field at the top boundary, i.e., $f\left(N^{2}\right) \equiv E_{y, t o p}$ with $N\left(=\beta / K_{o}\right)$, the modal index can be calculated. The dispersion relation

$$
f\left(N^{2}\right)=0
$$

corresponds to modal field solutions of (1). As noted in (2), $f$ is a single valued function of $\alpha_{p} \forall p$.

\section{B. The CI Method}

Having determined the dispersion relation, we outline the basic theory of determining the zeroes of the complex dispersion relation (4) and recommend improvements to assure the robustness and accuracy of the computation

1) Basic Theory: The CI method, originally stemming from complex analysis to determine the residues of a complex function [4] provides a powerful tool to find the complex valued roots of an algebraic equation. With the help of CI, one may first detect how many eigenvalues fall in a certain region of the complex $N^{2}$ plane.

As a consequence of Cauchy's Residue theorem, it follows that with the following contour integrals, the sum of the $k$-th powers of the encircled poles of $f$ can be evaluated, i.e.,

$$
I^{(k)}=\frac{1}{2 \pi j} \oint z^{k} \frac{\frac{d}{d z} f(z)}{f(z)} d z=\sum_{h=1}^{P} z_{h}^{k}, z \equiv N^{2} .
$$

Here, $P$ is the number of roots inside the contour and the summation runs over all encircled roots. As a consequence of (5), $I^{(0)}$ is the number of roots in the contour. Knowing the values for $I^{(0)} \ldots I^{(P)}$, an equivalent polynomial can be constructed [5] whose roots coincide with the encircled eigenvalues as follows:

$$
Q_{P}(z)=\sum_{k=0}^{P} c_{k} z^{k}
$$

where the values of the coefficients, and $c_{k}$ in (6) are given by the following recursion relation:

$$
\begin{aligned}
(P-k) c_{k}+I^{(1)} c_{1+k}+I^{(2)} c_{2+k} . .+I^{(P-k)} c_{P} & =0 \\
c_{P} & =1 .
\end{aligned}
$$

The roots of the polynomial can be determined by means of Laguerre's Method as recommended by, e.g., [5]. We found that a large number of roots inside the contour strongly affects the accuracy of the numerical evaluation of the roots, and for this reason, we usually divide the investigated part of the complex $N^{2}$ plane in subcontours, consisting of rectangles, containing not more than four roots. For convenience, the sides of the rectangle are chosen parallel to the real and imaginary $z$-axes.

2) Robust Evaluation of the CI: One of the most important aspects of getting accurate results is the proper evaluation of the integration in (5). The direct evaluation of (5) requires either the analytical determination of the derivative in the kernel or an efficient numerical scheme to evaluate the derivative in discrete points. Although $f\left(N^{2}\right)$ is an analytical function, for multilayered structures, it is time consuming to determine its derivative analytically. A practical way to overcome this difficulty would be to approximate the derivative with a numerical scheme, for example with a four-point difference operator. This scheme, however, is slowly converging if the function $f$ is strongly oscillating, which is indeed the case in the effective index regime in the vicinity of roots corresponding to higher order eigenvalues. The order of magnitude of the matrix elements may introduce another problem.

For these reasons, (5) is evaluated by introducing integration by parts, as follows:

$$
\begin{aligned}
& \int_{z_{1}}^{z_{2}} z^{k} \frac{f^{\prime}(z)}{f(z)} d z= \int_{z_{1}}^{z_{2}} z^{k} \frac{d}{d z} \ln (f(z)) d z \\
&= {\left[\{2 m \pi j+\ln (f)\} z^{k}\right]_{z_{1}}^{z_{2}} } \\
&-k \int_{z_{1}}^{z_{2}} z^{k-1} \ln (f) d z \\
&(k, m) \in \mathbf{Z} .
\end{aligned}
$$

Here, $z_{1}$ and $z_{2}$ indicate the segment in the complex $N^{2}$ plane, the principal branch of $\ln f$ must be taken, i.e., $-\pi<i m(\ln f)<\pi$, and $m$ indicates the true branch of $\ln f$, which can be obtained by keeping track of the number of times that $\operatorname{Im}\{\ln (f)\}$ crosses $\pi$ or $-\pi$.

The integration (8) is performed by means of Simpson's $2 / 3-4 / 3$ scheme [5], providing an accuracy proportional to the fourth power of the stepsize. Using (8), it is much simpler to evaluate the integral. However, the multivaluedness of the complex logarithm function must be taken care of. One must keep track of its several branches during evaluation. This procedure is called unwrapping the complex phase along the integration trajectory. It is done by inspecting, whether there is a jump from $\pi$ to $-\pi$, or vice versa, occurring in the value of the complex-valued logarithm function. Note that although on calculating the integral, the occurrence of extremely large numbers could be eliminated by introducing the complex logarithm function, these numbers indeed occur in the elements of the transfer matrices in (5). We can eliminate that problem 
by taking advantage of the asymptotic nature of hyperbolic functions

$$
\cosh (\Phi) \sim \sinh (\Phi) \sim \exp (\Phi) / 2
$$

for large values of $\Phi$. So for large values of $\pm \operatorname{Im}(\chi)$, with $\chi \equiv$ $\eta_{p} \alpha_{p} d_{p}$ for a given choice of the sign of $\alpha_{p}$, the corresponding transfer matrix can be approximated by

$$
\frac{\mp \exp \{\mp j \operatorname{Re}(\chi) \pm \operatorname{Im}(\chi)\}}{2}\left(\begin{array}{cc}
1 & \frac{-j}{\alpha_{p}} \\
j \alpha_{p} & 1
\end{array}\right) .
$$

Due to the logarithmic expressions in (8), the multiplication with the exponential expression in (10) need not to be evaluated.

3) Root Polishing: In principle, $\mathrm{CI}$ enables us to determine the roots with any desired accuracy but for numerical efficiency, we have chosen for a relatively large integration stepsize, typically, $\Delta N^{2}=10^{-5}$ followed by a sort of root polishing. The latter consists of a Newton-Raphson zero-finding procedure using the roots obtained from the rough integration as a starting point. The latter zero-finding procedure is terminated if the desired accuracy has been reached.

If one of those accurately determined roots would fall outside the subcontour, or if there are two or more roots coinciding, then the integration-polishing procedure is repeated using pole zero compensation as follows:

1) Subtracting

$$
\sum_{h=1}^{q} z_{h}^{k}
$$

from the first $P-q$ integrals $I^{(k)}$ defined in (8), where the summation of (11) runs over the $q$ "reliable" roots. This will result in the rough values for the yet undetermined roots.

2) Polishing using

$$
\frac{f(z)}{\prod_{h=1}^{q}\left(z-z_{h}\right)}
$$

instead of $f$.

If zeros are still found outside the subcontour, the CI is repeated with a smaller stepsize. The previous root-polishing and pole-zero compensation procedures have been found to be quite reliable.

\section{Evaluation OF RoOTS With CI}

On evaluating the roots, choices must be made with respect to the required accuracy, the definition of the contour, and the strength and thickness of PMLs, which will be discussed in the following.

With the aforementioned methods, the roots can be determined with large accuracy without much extra numerical effort. For this reason, the roots are usually evaluated with an inaccuracy corresponding $\Delta N \approx 10^{-9}$.

Next we will discuss the choice for the total contour for the CI. For nonactive structures, the square of the roots are known to be lying in the third and fourth quadrant of the complex $N^{2}$ plane, the $N$ values are chosen in, i.e, the fourth quadrant. The maximum value of $\operatorname{Re}\left(N^{2}\right)$ can be approximated by the fol- lowing. Multiplying (1) with $\eta E_{y}{ }^{*}(\eta=1-j \sigma)$, integrating the result over the cross section using partial integration leads to

$$
\int_{\text {comp.window }}\left\{\eta\left(k_{0}^{2} n^{2}-\beta^{2}\right)\left|E_{y}\right|^{2}-\frac{\left|\partial_{x} E_{y}\right|^{2}}{\eta}\right\} d x=0 .
$$

From this it follows $\max \left\{\operatorname{Re}\left(\eta_{\mathrm{p}} \mathrm{n}_{\mathrm{p}}^{2}\right)\right\}>\operatorname{Re}\left(\eta_{\mathrm{p}} \mathrm{N}^{2}\right)$ (see Appendix). Then, a safe integration boundary is $\operatorname{Re}\left(\mathrm{N}^{2}\right)<\max \left\{\operatorname{Re}\left(\eta_{\mathrm{p}} \mathrm{n}_{\mathrm{p}}^{2}\right)\right\} \equiv \mathrm{A}^{2}$, with $A$ real and positive. This conjecture is proven in the Appendix. Further, we may use the evanescent modes with a decay length much shorter than $L$, being a typical length of the structure (e.g., grating period) in the longitudinal direction may be neglected. So we require, i.e., $\operatorname{Im}(N)>-10 /\left(k_{0} L\right) \equiv-B$. A contour rectangle meeting the above requirements is given by

$$
\begin{aligned}
-B^{2} & <\operatorname{Re}\left(\mathrm{N}^{2}\right)<\mathrm{A}^{2} \\
-2 \sqrt{A^{2}+B^{2}} B & <\operatorname{Im}\left(\mathrm{N}^{2}\right)<0 .
\end{aligned}
$$

Simple rules for the choice of thickness and strength of PMLs are hard to formulate but a few guidelines can be given. The amplitude reflection coefficient at PML-non PML layer, both with index $n$, is

$$
r_{P M L}=\exp \left[-2 k_{0} \sqrt{N_{\mathrm{eff}}^{2}-n^{2}}(1-j \sigma) d_{P M L}\right] .
$$

For guided modes, where (14) should be understood as a ratio of increasing and decaying solutions, it can be seen that the modal field solutions are normally not affected by the PML if its thickness and strength $\sigma$ are not too small. With the latter, the reflection for radiation modes from the PML may also be small. However, $\left|r_{P M L}\right|$ tends to unity for grazing incidence, i.e., if $N_{\text {eff }} \approx n$. Then, in order for avoiding back reflections from PML to the central part of the structure the PML should be positioned "sufficiently far away."

\section{Determination of Field Profiles and InNer Product}

\section{A. Determination of Field Profiles}

After the modal indices have been determined accurately, the corresponding modal field $E_{y}(x)$ can be calculated by starting at the bottom with $E_{y, 1}=0$ and, i.e., $\partial_{x} E_{y, 1}=1$. Next, using (2), field and derivative at the other interfaces can be determined. The field at position $x$ inside a certain layer $p$ can now be calculated using (2) but with $d_{p}$ replaced by $x$.

\section{B. Determination of Inner Product}

To normalize fields properly as well as to get the eigenmode expansion, an inner product on the function space of the solutions of (1) must be determined. To do that, let us introduce the following variable:

$$
\xi \equiv \eta x
$$

with, as before, $\eta \equiv 1-j \hat{\sigma}$.

With this, (1) can be brought to the following form:

$$
\partial_{\xi \xi} E_{y}+\left(k_{0}^{2} n^{2}-\beta^{2}\right) E_{y}=0 .
$$


TABLE I

STRUCTURES IN CONSIDERATION IN THE CALCULATIONS

\begin{tabular}{l||l|l}
\hline & Structure 1 & Structure 2 \\
\hline \hline Free space wavelength & $1 \mu \mathrm{m}$ & $1 \mu \mathrm{m}$ \\
\hline Strength of PML $(\hat{\sigma})$ & 0.4 & 0.4 \\
\hline Thickness of PML & $1 \mu \mathrm{m}$ & $1 \mu \mathrm{m}$ \\
\hline $\mathrm{D}_{1,} \mathrm{n}_{1}$ & $5 \mu \mathrm{m}, 1.5$ & $2 \mu \mathrm{m}, 1.5$ \\
\hline $\mathrm{D}_{2,} \mathrm{n}_{2}$ & - & $1 \mu \mathrm{m}, 1.7$ \\
\hline $\mathrm{D}_{3,} \mathrm{n}_{3}$ & - & $2 \mu \mathrm{m}, 1.5$ \\
\hline $\mathrm{D}_{4,} \mathrm{n}_{4}$ & - & $1 \mu \mathrm{m}, 1.7$ \\
\hline $\mathrm{D}_{5,} \mathrm{n}_{5}$ & - & $2 \mu \mathrm{m}, 1.5$ \\
\hline
\end{tabular}

Equations (1) and (15b) are of Sturm-Liouville type [3], an equation whose eigenfunctions are known to form a complete set of functions. In particular, (15b) can be regarded as a scaled, one-dimensional Helmholtz equation, whose eigenfunctions are known to be orthogonal for the following scalar product:

$$
\int_{\text {c.w. }} E_{y}^{(n)}(\xi) E_{y}^{(m)}(\xi) d \xi=\int_{\text {c.w. }} \eta E_{y}^{(n)}(x) E_{y}^{(m)}(x) d x
$$

where the integration must be evaluated in the computational window (c.w.) defined by the two electric walls as depicted in Fig. 1 and the superscripts $(n)$ and $(m)$ are mode indices.

\section{RESUlTS AND DISCUSSION}

In this section, we will discuss results of the presented MEM applied to two structures given in Table I. The following will be checked:

- robustness, with respect to finding all modes in the considered $N^{2}$ rectangle;

- finding quasidegenerate modes, for example, the first even and odd mode of a symmetric coupler with large separation;

- the performance of PML with emphasis on examining the reflection on the dielectric interface of PML-non-PML dielectric interface;

- correct propagation.

Also, we will discuss in short the distribution of the modal indices in the $N$-plane, as given for the two structures in Fig. 4.The modal indices of structure 1 are found to lie on a single branch, which can be proven to be given by

$$
\begin{aligned}
N_{m} & =\sqrt{n^{2}-m^{2} / \gamma^{2}}, m=1,2 \ldots, \\
\gamma & \equiv\left\{4(1-j \sigma) d_{P M L}+2 d_{2}\right\} / \lambda .
\end{aligned}
$$

Equation (17) follows from (1) to (4). Using these and also that there are no reflections at the PML-central layer interface the field at the top boundary is, neglecting a proportionality constant

$$
E_{y, t o p}=\exp (j \pi \gamma \alpha)-\exp (-j \pi \gamma \alpha)
$$

with $\alpha \equiv \sqrt{n^{2}-N^{2}}$. Then requiring zero field at the top (17) follows.

Comparison of the modes obtained by this analytic formula and by $\mathrm{CI}$ showed that in fact, all of the first 70 modes were

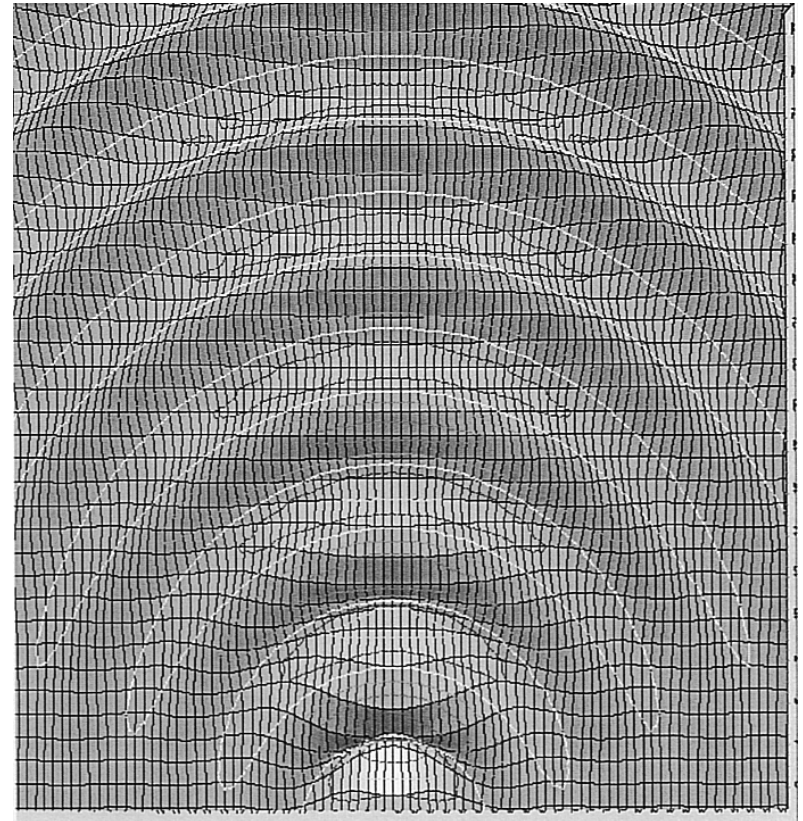

Fig. 2. Propagating a Gaussian beam along $z$ for the parameters of structure 1: results obtained by taking different numbers of eigenfunctions into account are compared to the exact analytical result.

found by the CI algorithm. For nonuniform structures like two, a distribution being similar to that of Structure 1 can be found, but different branches can be observed. For structure two consisting of two weakly guiding bimodal identical waveguides all guided system modes, virtually coinciding two by two, have been found. The indices of these are found to coincide with those of the open system as calculated with a standard mode solver.

In order to check correct propagation and performance of PML we have studied the propagation of a narrow Gaussian input beam given by

$$
E_{y}(x, z=0)=\frac{2}{\sqrt{\pi}} \exp \left(\frac{-20 x^{2}}{\mu m^{2}}\right)
$$

in Structure 1. We chose this simple structure to be able to compare the results of our numerical method with the exact analytical solution.

The previous field can be expanded in terms of the PML modes found with $\mathrm{C}$ I according to

$$
E_{y}(x, z=0)=\sum_{i=0}^{N} c_{i} \psi_{i}
$$

where $\psi_{i}$ 's are the normalized PML modes and coefficients $c_{i}$ are determined as follows:

$$
c_{i}=\int_{\text {comp.window }}(1-j \sigma) E_{y}(x, z=0) \psi_{i} d x .
$$

From Fig. 2 it can be seen, that propagation along $z$ leads to virtually no reflection from the PMLs.

We have also compared the results of the propagation with the analytical results. The latter follows from the Green's function approach as follows. Knowing, that the fundamental solution of 


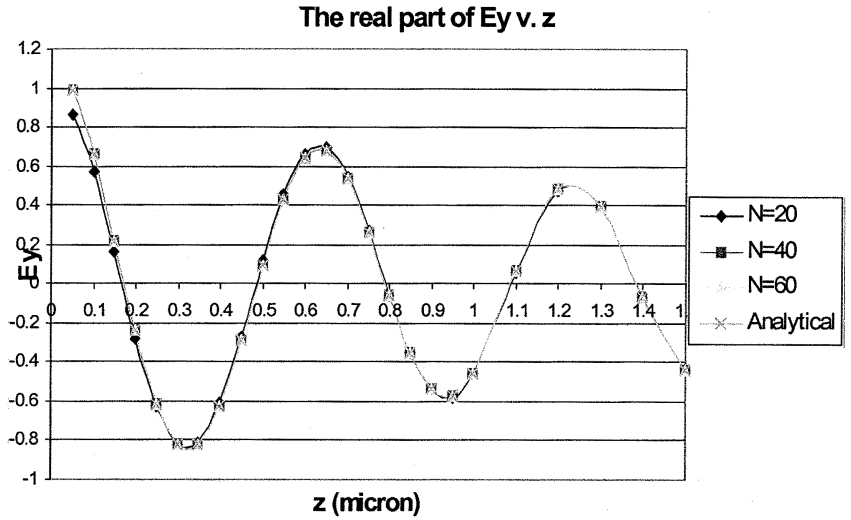

The imaginary part of Ey v. $z$

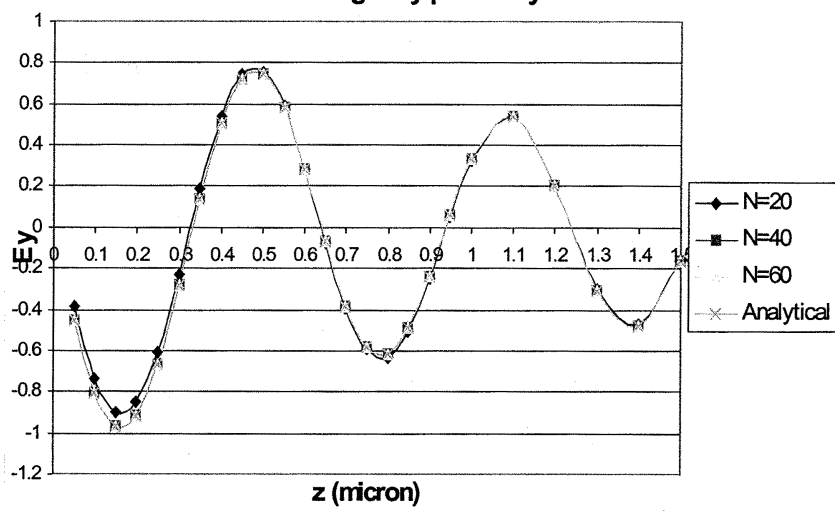

Fig. 3. Contour plot of the real part of $E_{y}$. Free-space wave propagation of a Gaussian beam defined by (18) in structure 1 (see table). Size of window: $-2.5 \mu<x<2.5 \mu, 0<z<5 \mu$.

the Helmholtz equation in the non PML part takes the following form [7]:

$$
G\left(\mathbf{r} \mid \mathbf{r}^{\prime}\right)=-\frac{j}{4} H_{o}^{(2)}\left(n k_{0} \sqrt{\left(x-x^{\prime}\right)^{2}+\left(z-z^{\prime}\right)^{2}}\right)
$$

by using the Method of Images [see, e.g., 7], the Green's Function being zero at $z^{\prime}=0$ can be constructed as follows:

$$
\begin{aligned}
\Gamma\left(\mathbf{r} \mid \mathbf{r}^{\prime}\right)=-\frac{j}{4} & {\left[H_{o}^{(2)}\left(n k_{0} \sqrt{\left(x-x^{\prime}\right)^{2}+\left(z-z^{\prime}\right)^{2}}\right)\right.} \\
& \left.-H_{o}^{(2)}\left(n k_{0} \sqrt{\left(x-x^{\prime}\right)^{2}+\left(z+z^{\prime}\right)^{2}}\right)\right]
\end{aligned}
$$

with $H_{0}^{(2)}(x)$ being the zeroth-order Hankel function of second kind. Thus, we obtain the following expression to determine the field distribution on the $(x-z)$ plane, given an input field:

$$
E_{y}(x, z)=\left.\int_{-\infty}^{\infty} E_{y}\left(x^{\prime}, z=0\right) \partial_{z^{\prime}} \Gamma\left(x, z, x^{\prime}, z^{\prime}\right)\right|_{z^{\prime}=0} d x^{\prime}
$$

where the integration must be evaluated for the same region as (20). These formulae were evaluated by means of the numerical engine of MAPLE.

From Fig. 3, it can be seen that the result of MEM coincides nicely with the exact results. The only exception is for small $z$ values where the MEM does not perform so well if a relatively

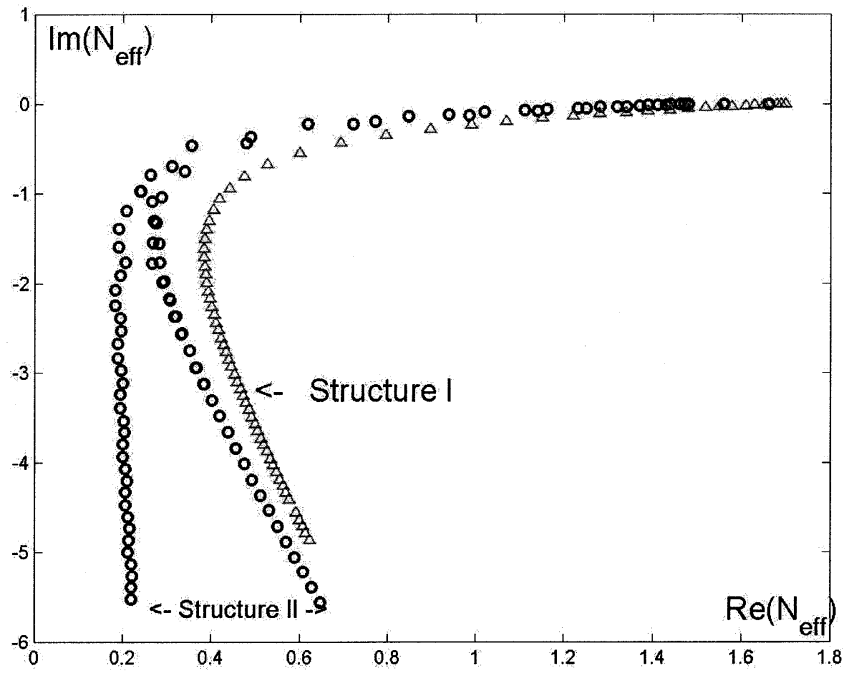

Fig. 4. Distributions of effective indices on the complex effective index plane.

low number of modes are taken into account. Here, modes corresponding to high spatial frequencies along $x$ play the some role, too. Since these modes are damped in the PML and rapidly radiated out in the open system, their role is minor for higher $z$ values. The behavior of Berenger and radiation modes is discussed by [8].

\section{CONCLUSION}

In this paper, a novel computational algorithm was described to determine the PML modes of stratified 2-D systems, closed with electric walls at the computational boundaries. It was found that with this new algorithm, the degenerate modes of a coupler, as well as higher order modes of several PML systems, could be found in a robust way. The properties of PML mode expansion were also investigated and it was found that our results with MEM coincide with the analytical results of an open system.

\section{APPENDIX}

Here we prove (13a) and (13b). From (13a), it follows that

$$
\operatorname{Re} \int_{\text {comp.window }}\left\{\eta\left(-k_{0}^{2} n^{2}+\beta^{2}\right)\left|E_{y}\right|^{2}\right\} d x<0 .
$$

Rearranging (A1), we get

$$
\begin{aligned}
& \operatorname{Re} \int_{\text {comp.window }}\left\{\eta N^{2}\left|E_{y}\right|^{2}\right\} d x \\
& <\operatorname{Re} \int_{\text {c.w. }}\left\{\eta \mathrm{n}^{2}\left|\mathrm{E}_{\mathrm{y}}\right|^{2}\right\} \mathrm{dx} \\
& <\max \left[\operatorname{Re} \int_{\text {c.w. }}\left\{\eta \mathrm{n}_{\mathrm{p}}^{2}\left|\mathrm{E}_{\mathrm{y}}\right|^{2}\right\} \mathrm{dx}\right] .
\end{aligned}
$$

From this it follows that

$$
\max \left\{\operatorname{Re}\left(\eta_{p} n_{p}^{2}\right)\right\}>\operatorname{Re}\left(\eta_{p} N^{2}\right) .
$$

Let us suppose that $\operatorname{Im}(\mathrm{N})<0, \operatorname{Re}\left(N^{2}\right)<A^{2}, \operatorname{Im}(N)<$ $B$. After some algebra. one can arrive to (13b). 


\section{ACKNOWLEDGMENT}

The authors would like to thank R. Stoffer and H. F. Bulthuis for helpful discussions and P. Bienstman for supplying computational results for testing purposes.

\section{REFERENCES}

[1] J. P. Berenger, "A perfectly matched layer for the absorption of electromagnetic waves," J. Comp. Phys., vol. 114, pp. 185-200, 1994.

[2] P. Bienstman, H. Derudder, R. Baets, F. Olyslager, and D. De Zutter, "Analysis of cylindrical waveguide discontinuities using vectorial eigenmodes and perfectly matched layers," IEEE Trans. Microwave Theory Technol., vol. 49, pp. 349-354, Feb. 2001.

[3] R. E. Smith et al., "Mode determination for planar waveguides using 4-Sheeted dispersion relation," IEEE J. Quantum Electron., vol. 28, pp. 1520-1526, June 1992.

[4] Morse and Fesbach, Methods of Theoretical Physics. New York: McGraw-Hill, 1978.

[5] Press et al., Numerical Recipes in C. New York: Cambridge Univ. Press, 1992.

[6] E. Anemogiannis et al., "Multilayer waveguides: Efficient numerical analysis of general structures," J. Lightwave Technol., vol. 10, pp. 1344-1351, Oct. 1992.

[7] L. B. Felsen and N. Markuwitz, Radiation and Scattering of Waves. New York: IEEE Press, 1994.

[8] P. Bienstman and R. Baets, "Advanced boundary conditions for eigenmode expansion models," J. Opt. Quantum Electron., vol. 34, pp. 523-540, May-June 2002.

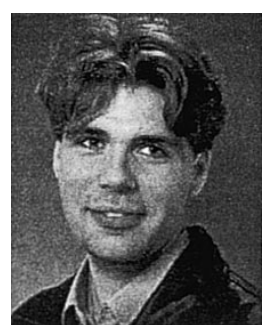

Szabolcs B. Gaál (M'98) was born in Budapest, Hungary, on June 7, 1971. He received the M.Sc. degree in electrical engineering from the Technical University of Budapest, Budapest, Hungary, in 1994 and the Ph.D. degree from the University of Twente, Twente, The Netherlands, in 2002.

From 1999 to 2000, he worked for the Hungarian Foreign Trade Bank, where he was responsible for developing risk management software. From 2000 to 2002, he was a Researcher at the University of Twente, where he elaborated on wave propagation algorithms to model the behavior of light in segmented waveguide sensors. Since 2002, he has been employed by Essent Energie, where his responsibility is to elaborate and implement algorithms to evaluate derivative contracts in the electric energy market. His research interests are in solution techniques of partial and stochastic differential equations of different types for different application areas.

Hugo J. W. M. Hoekstra, photograph and biography not available at the time of publication.

Paul V. Lambeck, photograph and biography not available at the time of publication. 